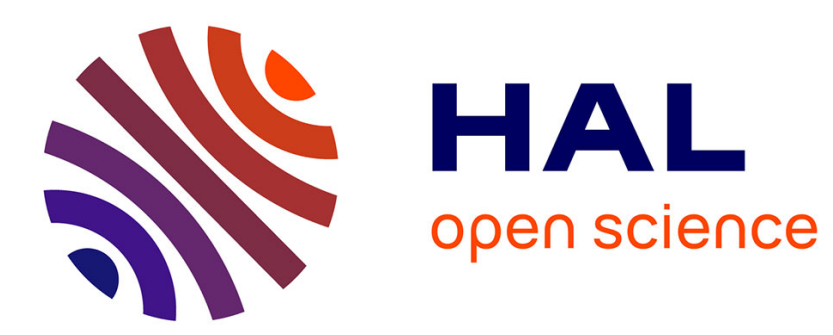

\title{
Exact correlation functions in an asymmetric exclusion model with open boundaries
}

\author{
B. Derrida, M. Evans
}

\section{To cite this version:}

B. Derrida, M. Evans. Exact correlation functions in an asymmetric exclusion model with open boundaries. Journal de Physique I, 1993, 3 (2), pp.311-322. 10.1051/jp1:1993132 . jpa-00246724

HAL Id: jpa-00246724

https://hal.science/jpa-00246724

Submitted on 1 Jan 1993

HAL is a multi-disciplinary open access archive for the deposit and dissemination of scientific research documents, whether they are published or not. The documents may come from teaching and research institutions in France or abroad, or from public or private research centers.
L'archive ouverte pluridisciplinaire HAL, est destinée au dépôt et à la diffusion de documents scientifiques de niveau recherche, publiés ou non, émanant des établissements d'enseignement et de recherche français ou étrangers, des laboratoires publics ou privés. 
Classification

Physics Abstracts

$05.40-05.50-05.60$

\title{
Exact correlation functions in an asymmetric exclusion model with open boundaries
}

\author{
B. Derrida and M.R. Evans \\ Service de Physique Théorique ${ }^{1}$, C.E. Saclay, 91191 Gif-sur-Yvette Cedex, France \\ Received 6 June 1992, accepted 1st July, 1992
}

\begin{abstract}
Résumé. - Nous considérons un modèle d'exclusion asymétrique pour lequel des particules avec des interactions de coeur dur sautent avec une direction privilégiée. Dans le cas de conditions de bord ouvertes, la valeur moyenne des nombres d'occupation était déjà connue. Nous généralisons ces expressions à toutes les fonctions de corrélation. Nous étudions plusieurs limites continues de la fonction à deux points et montrons que les conditions de bord produisent des corrélations à longue portée. Nous discutons aussi la présence de ces corrélations à longue portée dans un modèle de croissance relié,
\end{abstract}

\begin{abstract}
Asymmetric exclusion models are systems of particles hopping in a preferred direction with hard core interactions. Exact expressions for the average occupations have previously been derived in the one dimensional fully asymmetric case with open boundaries. Generalisations of these expressions are presented for all correlation functions. We discuss several continuous limits of the two-point correlation functions and show that due to the boundary conditions, long range correlations persist in the bulk. We also discuss the presence of these long range correlations in a related growth model.
\end{abstract}

\section{Introduction.}

Driven diffusive lattice gas systems are known as non-equilibrium models which have interesting stationary states [1-9]. They belong to a broader class of systems, including growth processes [10-12] and models exhibiting self-organised criticality [13,14], which evolve according to microscopic dynamical rules that are local and stochastic. The absence of detailed balance conditions for the dynamics of these models implies that their steady states usually cannot be described as the Gibbs measure associated with any reasonable energy function. It is expected that such systems should, in general, present long range correlations in their steady state $[13,15,16]$.

$\left({ }^{1}\right)$ Laboratoire de la Direction des Sciences de la Matière du Commissariat à l'Energie Atomique. 
In the present paper, we report some exact results on the correlations functions of an asymmetric one dimensional exclusion model [8], which is a particularly simple example of a driven lattice gas. For this system, particles are chosen for update at random and can then jump to their neighbouring site to the right, only when this site is not occupied. This model has been considered recently by various authors in different geometries $[10,6,7,8]$. The simplest case is that of periodic boundary conditions, where the dynamics conserve the number of particles in the system. It has been shown $[10,17]$ that steady states correspond to having an equal weight for all possible configurations (with the right number of particles). Thus for periodic boundary conditions, the occupation variables of the different sites have no correlation and the correlation functions are trivial. The time dependence is more complicated, nevertheless the eigenvalues of the Master equation can be computed by the Bethe ansatz method $[17,9]$. Another situation which has recently been considered [7] is that of the system with periodic boundary conditions, but with a blockage inserted to break the translational invariance. The blockage takes the form of a special bond where the hopping rate differs from the value it takes for the other bonds. The presence of this single impurity, which can be thought of as a kind of boundary condition, is sufficient to create long range correlations in the system.

The case of open boundary conditions where particles enter at the left end and leave at the right end has also been studied $[18,6,8]$, and is the situation we consider here. Again, the effect of the boundary conditions is to give rise to power law decays in the density profile (the time average of the occupancy as a function of the position along the chain).

The model we consider, which is exactly the same as in [8], is defined as follows: each site $i(1 \leq i \leq N)$ of a one dimensional lattice of $N$ sites is either occupied by a particle $\left(\tau_{i}=1\right)$ or empty $\left(\tau_{i}=0\right)$. The evolution of this system is governed by the following rule: at each time step $t \rightarrow t+1$, an integer $i$ is chosen at random between 0 and $N$ (i.e. the probability of choosing $i$ is $1 /(N+1)$ ). If the integer $i$ is between 1 and $N-1$, the particle on site $i$ (if there is one) jumps to site $i+1$ (if this site is empty), i.e.

$$
\begin{array}{r}
\tau_{i}(t+1)=\tau_{i+1}(t) \tau_{i}(t) \\
\tau_{i+1}(t+1)=\tau_{i+1}(t)+\left(1-\tau_{i+1}(t)\right) \tau_{i}(t)
\end{array}
$$

If the integer chosen is $i=0$, then if site 1 is empty it becomes occupied with probability $\alpha$, whereas if it is occupied it remains occupied. Similarly, if the integer chosen is $i=N$, then if site $N$ is occupied it becomes empty with probability $\beta$ whereas if it is empty it remains empty. The values of $\alpha$ and $\beta$ define the (open) boundary conditions and phase transitions can occur as $\alpha$ and $\beta$ are varied $[6,8]$.

The exact solution of this model [8] was obtained by writing a recursion which gives the steady state of a lattice of $N$ sites in terms of the steady state for a lattice of $N-1$ sites. This recursion is recalled in the Appendix, however, it is rather complicated and the calculation of observables like correlation functions is not straightforward. In reference [8], the expression for the density profile $\left\langle\tau_{i}\right\rangle_{N}$ (i.e. the one point correlation function) for all system sizes $N$, was derived in the case

$$
\alpha=\beta=1 \text {. }
$$

In this case (and more generally when $\alpha=\beta \leq 1$ ) one can show that the dynamics of particles moving to the right is identical to that of holes moving to the left. This particle-hole symmetry implies that in the steady state

$$
\left\langle\tau_{i_{1}} . \quad \tau_{i_{n}}\right\rangle_{N}=\left\langle\left(1-\tau_{N+1-i_{1}}\right) . \quad\left(1-\tau_{N+1-i_{n}}\right)\right\rangle_{N},
$$


where $(. .\rangle_{N}$ indicates an average in the steady state of a system of size $N$.

The main purpose of this paper is to present, for the same values of $\alpha$ and $\beta$ equal to one, a similar result for the two point correlation function, to that of [8] for the one point correlation function, and to propose a conjecture (that we checked on the computer) for the three and the four point functions. A main consequence of our results is that for this exactly soluble model, we can show the existence of long range correlations.

The paper is organised as follows. In section 2, we recall the exact expression of the one point correlation function [8], and we give our main results: the exact expressions for the pair correlation functions and conjectures (that we checked on the computer) for the three point and four point correlations. In section 3 , we obtain the expression of $\left\langle\tau_{i} \tau_{j}\right\rangle_{N}$ in two continuous limits of this problem: firstly, the boundary region, for which $N$ becomes infinite first, and then $\left\langle\tau_{i} \tau_{j}\right\rangle_{N}$ is estimated for $i$ and $j$ large; secondly, the bulk of the chain, where $N$ becomes large with $i=N x$ and $j=N y, x$ and $y$ being fixed. In section 4, we use the equivalence of this one dimensional exclusion problem with that of a growing interface to interpret our results in terms of height variables.

\section{Exact expressions of the correlation functions.}

The key to obtaining exact expressions correlation functions is to consider the steady state of the weights $f_{N}\left(\tau_{1}, \ldots \tau_{N}\right)$ of occupancy configurations $\left\{\tau_{1}, . \tau_{N}\right\}$. The stationary probability $p_{N}\left(\tau_{1}, . \quad \tau_{N}\right)$ of an occupancy configuration is then given by

$$
p_{N}\left(\tau_{1}, . \quad \tau_{N}\right)=\frac{f_{N}\left(\tau_{1}, \ldots \tau_{N}\right)}{\sum_{\tau_{1}=1,0} \cdot \sum_{\tau_{N}=1,0} f_{N}\left(\tau_{1}, . \tau_{N}\right)} .
$$

The steady state of the weights may be written in the form of a recursion [8] which we provide in the Appendix. In principle the knowledge of the correlations of arbitrary order $n$ follows by computing the correlation functions

$$
\left\langle\tau_{i_{1}} \tau_{i_{2}} . \quad \tau_{i_{n}}\right\rangle_{N}=\frac{\sum_{\tau_{1}=1,0} \ldots \sum_{\tau_{N}=1,0} \tau_{i_{1}} \tau_{i_{2}} \ldots \tau_{i_{n}} f_{N}\left(\tau_{1}, \ldots \tau_{N}\right)}{\sum_{\tau_{1}=1,0} \cdot \sum_{\tau_{N}=1,0} f_{N}\left(\tau_{1}, \cdot \tau_{N}\right)}
$$

however the recursion is sufficiently complicated that the way to do this calculation is not immediately obvious.

The 1-point correlation function $\left\langle\tau_{i}\right\rangle_{N}$ was obtained from a generating function formed by summing over indices $i$ and $N$ [8]. The result could be written in two forms: as a sum

$$
\left\langle\tau_{k}\right\rangle_{N}=\frac{1}{A(N+1)} \sum_{p=0}^{N-k} A(p) A(N-p)
$$

where

$$
A(m)=\frac{(2 m) !}{m !(m+1) !}
$$

or alternatively as a closed expression

$$
\left\langle\tau_{k}\right\rangle_{N}=\frac{1}{2}+\frac{1}{4} \frac{(2 k) !(N !)^{2}(2 N-2 k+2) !}{(k !)^{2}(2 N+1) ![(N-k+1) !]^{2}}(N-2 k+1) .
$$

We have followed the same generating function approach to obtain the 2-point correlation functions. However, because the derivation is rather tortuous and gives no physical insight 
into the problem we do not present it here. The result we obtained is

$$
\left\langle\tau_{k_{1}} \tau_{k_{2}}\right\rangle_{N}=\frac{1}{A(N+1)} \sum_{p_{1}=0}^{N-k_{1}} A\left(p_{1}\right) \sum_{p_{2}=0}^{N-1-k_{2}-p_{1}} A\left(p_{2}\right) A\left(N-1-p_{1}-p_{2}\right)
$$

where $k_{1}>k_{2}$.

We checked this expression against a direct evaluation of (5) on the computer for system sizes up to $N=10$ and found a perfect agreement. On comparing the expressions (6) and (9) for $\left\langle\tau_{k}\right\rangle$ and $\left\langle\tau_{k_{1}} \tau_{k_{2}}\right\rangle$, we conjectured the following expressions for higher order correlations

$$
\begin{aligned}
\left\langle\tau_{k_{1}} \tau_{k_{2}} \tau_{k_{3}}\right\rangle_{N}= & \frac{1}{A(N+1)} \sum_{p_{1}=0}^{N-k_{1}} A\left(p_{1}\right) \sum_{p_{2}=0}^{N-1-k_{2}-p_{1}} A\left(p_{2}\right) \\
& \times \sum_{p_{3}=0}^{N-2-k_{3}-p_{1}-p_{2}} A\left(p_{3}\right) A\left(N-2-p_{1}-p_{2}-p_{3}\right)
\end{aligned}
$$

where $k_{1}>k_{2}>k_{3}$, and

$$
\begin{aligned}
& \left\langle\tau_{k_{1}} \tau_{k_{2}} \tau_{k_{3}} \tau_{k_{4}}\right\rangle_{N}=\frac{1}{A(N+1)} \sum_{p_{1}=0}^{N-k_{1}} A\left(p_{1}\right) \sum_{p_{2}=0}^{N-1-k_{2}-p_{1}} A\left(p_{2}\right) \\
& \times \sum_{p_{3}=0}^{N-2-k_{3}-p_{1}-p_{2}} A\left(p_{3}\right)^{N-3-k_{4}-p_{1}-p_{2}-p_{3}} \sum_{p_{4}=0}^{N} A\left(p_{4}\right) A\left(N-3-p_{1}-p_{2}-p_{3}-p_{4}\right)
\end{aligned}
$$

where $k_{1}>k_{2}>k_{3}>k_{4}$.

As before we checked these conjectures against the computer and found that they are indeed in excellent agreement.

A convenient way of writing $(9,10,11)$ is in a form similar to $(6)$

$$
\begin{aligned}
\left\langle\tau_{k_{1}} \tau_{k_{2}}\right\rangle_{N} & =\frac{1}{A(N+1)} \sum_{q=k_{1}}^{N} A(q) A(N-q)\left\langle\tau_{k_{2}}\right\rangle_{q-1} \\
\left\langle\tau_{k_{1}} \tau_{k_{2}} \tau_{k_{3}}\right\rangle_{N} & =\frac{1}{A(N+1)} \sum_{q=k_{1}}^{N} A(q) A(N-q)\left\langle\tau_{k_{2}} \tau_{k_{3}}\right\rangle_{q-1} \\
\left\langle\tau_{k_{1}} \tau_{k_{2}} \tau_{k_{3}} \tau_{k_{4}}\right\rangle_{N} & =\frac{1}{A(N+1)} \sum_{q=k_{1}}^{N} A(q) A(N-q)\left\langle\tau_{k_{2}} \tau_{k_{3}} \tau_{k_{4}}\right\rangle_{q-1}
\end{aligned}
$$

and we believe that all other correlation functions are similarly given by

$$
\left\langle\tau_{k_{1}} \tau_{k_{2}} . \quad \tau_{k_{n}}\right\rangle_{N}=\frac{1}{A(N+1)} \sum_{q=k_{1}}^{N} A(q) A(N-q)\left\langle\tau_{k_{2}} . \quad \tau_{k_{n}}\right\rangle_{q-1} \text { where } k_{1}>k_{2} . \quad>k_{n} .
$$

We feel that there should be a physical reason for (15) (that would in turn furnish a more direct derivation for the solution of the model ) although we could not think of it.

A simple relationship between the 1 and 2 point correlation functions was derived in [8]:

$$
\left\langle\tau_{k}\right\rangle_{N}-\left\langle\tau_{k+1} \tau_{k}\right\rangle_{N}=\left\langle\tau_{N}\right\rangle_{N} \text { where } 1 \leq k \leq N-1
$$


This relationship expresses a conserved current of particles along the chain. To see that (6) and (8) satisfy (16), we rewrite (6) as

$$
\left\langle\tau_{k}\right\rangle_{N}=\left\langle\tau_{k+1}\right\rangle_{N}+\frac{A(k) A(N-k)}{A(N+1)}
$$

and $(9)$ as

$$
\begin{aligned}
\left\langle\tau_{k+1} \tau_{k}\right\rangle_{N} & =\left\langle\tau_{k+2} \tau_{k+1}\right\rangle_{N}+\frac{A(k)}{A(N+1)} \sum_{p=0}^{N-k-1} A(p) A(N-1-k-p) \\
& =\left\langle\tau_{k+2} \tau_{k+1}\right\rangle_{N}+\frac{A(k) A(N-k)}{A(N+1)}
\end{aligned}
$$

where we have used the identity

$$
\sum_{m=0}^{N} A(m) A(N-m)=A(N+1)
$$

which follows from the fact that

$$
\sum_{m=0}^{\infty} A(m) x^{m}=\frac{1-\sqrt{1-4 x}}{2 x}
$$

Subtracting (19) from (17) then gives

$$
\left\langle\tau_{k}\right\rangle_{N}-\left\langle\tau_{k+1} \tau_{k}\right\rangle_{N}=\left\langle\tau_{k+1}\right\rangle_{N}-\left\langle\tau_{k+2} \tau_{k+1}\right\rangle_{N} \quad \text { where } 1 \leq k \leq N-2 .
$$

At the right hand boundary $\left\langle\tau_{N-1}\right\rangle_{N},\left\langle\tau_{N} \tau_{N-1}\right\rangle_{N}$ have the simple expressions (17),(9)

$$
\begin{aligned}
\left\langle\tau_{N-1}\right\rangle_{N} & =\left\langle\tau_{N}\right\rangle_{N}+\frac{A(N-1)}{A(N+1)} \\
\left\langle\tau_{N} \tau_{N-1}\right\rangle_{N} & =\frac{A(N-1)}{A(N+1)}
\end{aligned}
$$

so that (16) is satisfied when $k=N-1$. Application of (22) then implies that (16) is satisfied for $1 \leq k \leq N-1$.

From now on we will focus on the 2-point correlation functions. From these we can calculate the fluctuations in the total number of particles $M$ in the system, which is given by

$$
M=\sum_{k=1}^{N} \tau_{k} .
$$

The average $\langle M\rangle_{N}$ is equal to $N / 2$ because of the particle-hole symmetry in the problem which implies from (3)

$$
\left\langle\tau_{i}\right\rangle_{N}=1-\left\langle\tau_{N+1-i}\right\rangle_{N}
$$

The mean square particle number is given by

$$
\left\langle M^{2}\right\rangle_{N}=\frac{N}{2}+2 \sum_{j>k}\left\langle\tau_{j} \tau_{k}\right\rangle_{N}
$$


The second term in this expression may be evaluated by using (9) and rearranging the sums to give

$$
\begin{aligned}
& \sum_{j>k}\left\langle\tau_{j} \tau_{k}\right\rangle_{N} \\
& =\frac{1}{A(N+1)} \sum_{k=1}^{N-1} \sum_{j=k+1}^{N} \sum_{p_{1}=0}^{N-j} A\left(p_{1}\right) \sum_{p_{2}=0}^{N-k-1-p_{1}} A\left(p_{2}\right) A\left(N-1-p_{1}-p_{2}\right) \\
& =\frac{1}{A(N+1)} \sum_{p_{1}=0}^{N-2} A\left(p_{1}\right) \sum_{p_{2}=0}^{N-p_{1}-2} \frac{\left(N-p_{1}-p_{2}-1\right)\left(N-p_{1}+p_{2}\right)}{2} A\left(p_{2}\right) A\left(N-1-p_{1}-p_{2}\right) . \\
& =\frac{1}{A(N+1)}\left\{\sum_{p_{1}=0}^{N-2} A\left(p_{1}\right) \sum_{p_{2}=0}^{N-2-p_{1}} A\left(p_{2}\right) A\left(N-1-p_{1}-p_{2}\right) \frac{\left(N-p_{1}-1\right)\left(N-p_{1}\right)}{2}\right. \\
& \left.-\sum_{p_{1}=0}^{N-2} A\left(p_{1}\right) \sum_{p_{2}=0}^{N-2-p_{1}} \frac{\left(p_{2}+1\right) p_{2}}{2} A\left(p_{2}\right) A\left(N-1-p_{1}-p_{2}\right)\right\}(28)
\end{aligned}
$$

The labels $p_{1}, p_{2}$ may be interchanged in the second term on the r.h.s. of (28) which allows the two terms in (28) to be combined to give

$$
\sum_{j>k}\left\langle\tau_{j} \tau_{k}\right\rangle_{N}=\frac{1}{A(N+1)} \sum_{p_{1}=0}^{N-2} N\left[\frac{N-2 p_{1}-1}{2}\right] A\left(p_{1}\right) \sum_{p_{2}=0}^{N-p_{1}-2} A\left(p_{2}\right) A\left(N-1-p_{1}-p_{2}\right) .
$$

Use of the identity (20) and

$$
\sum_{m=0}^{N} m A(m) A(N-m)=\frac{N}{2} A(N+1)
$$

which follows from (7) leads to

$$
\sum_{j>k}\left\langle\tau_{j} \tau_{k}\right\rangle_{N}=-\frac{N}{2}+\frac{N(N+1)}{2} \frac{A(N)}{A(N+1)}
$$

We then obtain

$$
\begin{aligned}
\left\langle M^{2}\right\rangle_{N} & =\frac{N\left(N^{2}+N+1\right)}{2(2 N+1)} \\
\left\langle M^{2}\right\rangle_{N}-\langle M\rangle_{N}^{2} & =\frac{N(N+2)}{4(2 N+1)} .
\end{aligned}
$$

In the limit of large $N$ the leading behaviour of $\left\langle M^{2}\right\rangle_{N}-\langle M\rangle_{N}^{2}$ is $N / 8$. This is to be compared with the value $N / 4$ that would be obtained for the leading behaviour if correlations were ignored. Clearly, long range correlations due to the presence of the boundaries have a significant effect in reducing the fluctuations of $M$. Our aim in the following sections will be to analyse in more detail the form of these correlations in the large $N$ limit.

\section{The continuous limit.}

For large system sizes, there are two simple regimes one may consider for the density profile $\left\langle\tau_{i}\right\rangle_{N}$, which is the average occupation number as a function of position along the chain. Firstly 
we have the boundary regions where the profile decays rapidly from the boundary values. Secondly there is the bulk where the density profile approaches a plateau with corrections to occupancy $1 / 2$ that follow a power law in $N$ [6]. An analytic form for the density profile has been obtained in these two regimes [8] by considering two continuous limits of (8). For the boundary region one lets $N \rightarrow \infty$ first and then lets the distance from the boundary become large. For the bulk [19] one considers distances from the boundary that are proportional to $N$ and lets $N \rightarrow \infty$. A result of [8] was that in the bulk the corrections to the occupancy $1 / 2$ are $N^{-1 / 2}$ rather than $N^{-1}$ as would be predicted by the mean field theory $[6,8]$. This suggests that the effect of the boundary conditions extends beyond the boundary region into the bulk. In the present section we shall repeat this kind of analysis for the 2-point correlation functions.

\section{The boundary region.}

For the boundary region we consider sites at a fixed position relative to the left hand boundary and let $N \rightarrow \infty$. The behaviour at sites near the right hand boundary can be related to the behaviour near the left hand boundary via the particle-hole symmetry (3) which implies

$$
\begin{aligned}
\left\langle\tau_{k_{1}} \tau_{k_{2}}\right\rangle_{N}-\left\langle\tau_{k_{1}}\right\rangle_{N}\left\langle\tau_{k_{2}}\right\rangle_{N} & =\left\langle\tau_{N+1-k_{2}} \tau_{N+1-k_{1}}\right\rangle_{N}-\left(\tau_{N+1-k_{2}}\right\rangle_{N}\left\langle\tau_{N+1-k_{1}}\right\rangle_{N} \\
& =\left\langle\tau_{N+1-k_{2}} \tau_{N+1-k_{2}}\right\rangle_{N}-\left(1-\left\langle\tau_{k_{1}}\right\rangle_{N}\right)\left(1-\left\langle\tau_{k_{2}}\right\rangle_{N}\right) .
\end{aligned}
$$

In the following we shall make use of Stirling's approximation which determines the large $N$ behaviour of $A(N)$ as

$$
A(N)=\frac{1}{\sqrt{\pi}} \frac{4^{N}}{N^{3 / 2}}\left[1+O\left(N^{-1}\right)\right]
$$

On letting $N \rightarrow \infty$ in (9), $k_{1}$ and $k_{2}$ being fixed with $k_{1}>k_{2}$, we find

$$
\left\langle\tau_{N+1-k_{1}} \tau_{N+1-k_{2}}\right\rangle_{N} \simeq \sum_{p_{1}=0}^{k_{2}-1} \frac{A\left(p_{1}\right)}{4^{p_{1}+1}} \sum_{p_{2}=0}^{k_{1}-1-p_{1}} \frac{A\left(p_{2}\right)}{4^{p_{2}+1}}
$$

We now assume $k_{1}, k_{2}, k_{1}-k_{2}$ are large so that we may replace $k_{2}-1$ by $k_{2}$ and $k_{1}-1$ by $k_{1}$ on the right hand side of (37), and use

$$
\sum_{p=0}^{m} \frac{A(p)}{4^{p+1}} \simeq \frac{1}{2}-\frac{1}{2 \sqrt{\pi m}} \text { for large } m
$$

to give

$$
\left\langle\tau_{N+1-k_{2}} \tau_{N+1-k_{1}}\right\rangle_{N} \simeq \frac{1}{4}-\frac{1}{4 \sqrt{\pi k_{2}}}-\sum_{p_{1}=0}^{k_{2}} \frac{A\left(p_{1}\right)}{4^{p_{1}+1}} \frac{1}{2 \sqrt{\pi\left(k_{1}-p_{1}\right)}} .
$$

The sum in (39) may be written as

$$
\sum_{p_{1}=0}^{k_{2}} \frac{A\left(p_{1}\right)}{4^{p_{1}+1}} \frac{1}{2 \sqrt{\pi\left(k_{1}-p_{1}\right)}}=\sum_{p_{1}=0}^{k_{2}} \frac{A\left(p_{1}\right)}{2 \sqrt{\pi} 4^{p_{1}+1}}\left[\frac{1}{\left(k_{1}-p_{1}\right)^{1 / 2}}-\frac{1}{k_{1}^{1 / 2}}\right]+\sum_{p_{1}=0}^{k_{2}} \frac{A\left(p_{1}\right)}{2 \sqrt{\pi} 4^{p_{1}+1} k_{1}^{1 / 2}}
$$

for which the first term may be cvaluated as an integral, after using (36), and the second term is given by (38). Eventually we find

$$
\left(\tau_{N+1-k_{1}} \tau_{N+1-k_{2}}\right\rangle_{N} \simeq \frac{1}{4}-\frac{1}{4 \pi}\left(\frac{1}{k_{1}^{1 / 2}}+\frac{1}{k_{2}^{1 / 2}}\right)+\frac{1}{4 \pi} \frac{\left(k_{1}-k_{2}\right)^{1 / 2}}{k_{1} k_{2}^{1 / 2}}
$$


The average occupancy in the boundary region is given from (8) when $N \rightarrow \infty$ by

$$
\left\langle\tau_{k}\right\rangle_{\infty} \simeq \frac{1}{2}+\frac{1}{2 \sqrt{\pi k}}+O\left(k^{-3 / 2}\right)
$$

so that as $N \rightarrow \infty$ the connected correlation (35) becomes

$$
\left\langle\tau_{k_{1}} \tau_{k_{2}}\right\rangle_{\infty}-\left\langle\tau_{k_{1}}\right\rangle_{\infty}\left\langle\tau_{k_{2}}\right\rangle_{\infty} \simeq \frac{-1}{4 \pi\left(k_{1} k_{2}\right)^{1 / 2}}\left[1-\left(1-\frac{k_{2}}{k_{1}}\right)^{1 / 2}\right]
$$

It is worth noting that expression (43), which was obtained for $k_{1} \gg 1, k_{2} \gg 1, k_{1}-k_{2} \gg 1$, remains valid when $k_{1}-k_{2}=1$. This may be checked by making use of the relationship (16) in the limit $N \rightarrow \infty$.

The bulk.

To take the continuous limit of (12) in the bulk, we will make $N$ large keeping the ratios $k_{1} / N$ and $k_{2} / N$ fixed:

$$
k_{1}=N y, k_{2}=N x, \text { where } k_{1}>k_{2}, y>x .
$$

We first rewrite $(12)$ as

$$
\begin{aligned}
\left\langle\tau_{k_{1}} \tau_{k_{2}}\right\rangle_{N} & =\left\langle\tau_{k_{2}+1} \tau_{k_{2}}\right\rangle_{N}-\sum_{q=0}^{k_{1}-k_{2}-2} \frac{A\left(N-1-k_{2}-q\right) A\left(k_{2}+1+q\right)}{A(N+1)}\left\langle\tau_{k_{2}}\right\rangle_{k_{2}+q} \\
& =\left\langle\tau_{k_{2}}\right\rangle_{N}-\left\langle\tau_{N}\right\rangle_{N}-\sum_{q=0}^{k_{1}-k_{2}-2} \frac{A\left(N-1-k_{2}-q\right) A\left(k_{2}+1+q\right)}{A(N+1)}\left\langle\tau_{k_{2}}\right\rangle_{k_{2}+q}
\end{aligned}
$$

In going from (45) to (46), we have used the relationship (16). The mean occupancy in the bulk is given from (8) (in the limit defined by (44) and $N \rightarrow \infty$ ) as

$$
\left\langle\tau_{N x}\right\rangle_{N}=\frac{1}{2}+\frac{1}{2 \sqrt{\pi} N^{1 / 2}} \frac{1-2 x}{[x(1-x)]^{1 / 2}}+O\left(N^{-3 / 2}\right),
$$

whereas the mean occupancy on the right hand boundary is given from (6) as

$$
\left\langle\tau_{N}\right\rangle_{N}=\frac{N+2}{2(2 N+1)}=\frac{1}{4}+\frac{3}{8 N}+O\left(N^{-2}\right) .
$$

On inserting $(36,44,47,48)$ into $(46)$ we have

$$
\begin{aligned}
& \left\langle\tau_{N y} \tau_{N x}\right\rangle_{N}=\frac{1}{4}+\frac{1}{2 \sqrt{\pi} N^{1 / 2}} \frac{1-2 x}{[x(1-x)]^{1 / 2}}-\frac{3}{8 N} \\
& -\frac{1}{4 \sqrt{\pi} N^{3 / 2}} \sum_{q=0}^{N(y-x)-2} \frac{1}{(1-x-z)^{3 / 2}(x+z)^{3 / 2}}\left\langle\tau_{N x}\right\rangle_{N(x+z)}+O\left(N^{-3 / 2}\right),
\end{aligned}
$$

where

$$
q=N z \text {. }
$$

We now make use of the bulk expression (47) for the average occupancy in the final term of (49). At first glance this does not appear justified for the small values of $q$ in the sum. However 
the error produced by using the bulk expression (47) in these terms is $O\left(N^{-3 / 2}\right)$. Thus, as long as we are ultimately interested only in orders up to $O\left(N^{-1}\right)$, this substitution is valid and we find, after replacing the sums by integrals,

$$
\begin{aligned}
& \left\langle\tau_{N y} \tau_{N x}\right\rangle_{N}= \\
& \quad \frac{1}{4}+\frac{1}{N^{1 / 2}}\left\{\frac{1}{2 \sqrt{\pi}} \frac{1-2 x}{[x(1-x)]^{1 / 2}}-\frac{1}{8 \sqrt{\pi}} \int_{0}^{y-x} \frac{d z}{(1-x-z)^{3 / 2}(x+z)^{3 / 2}}\right\} \\
& \quad+\frac{1}{N}\left\{-\frac{3}{8}+\frac{1}{8 \pi} \int_{0}^{y-x} \frac{d z}{(1-x-z)^{3 / 2}(x+z)^{3 / 2}} \frac{x-z}{[x z(x+z)]^{1 / 2}}\right\}+O\left(N^{-3 / 2}\right) .
\end{aligned}
$$

The integrals in (51) may be evaluated and one finds that within the connected correlation the terms up to order $O\left(N^{-1 / 2}\right)$ in (51) are cancelled. The leading term for the connected correlation is then given (for $y>x$ ) by

$$
\begin{aligned}
& \left\langle\tau_{N y} \tau_{N x}\right\rangle_{N}-\left\langle\tau_{N y}\right\rangle\left\langle\tau_{N x}\right\rangle_{N}= \\
& \quad \frac{1}{N}\left\{\frac{3}{4 \pi} \arctan \left[\left(\frac{y-x}{x(1-y)}\right)^{1 / 2}\right]-\frac{3}{8}-\frac{1}{4 \pi}\left(\frac{y-x}{x(1-y)}\right)^{1 / 2}\left[1-\frac{x}{1-x}-\frac{1-y}{y}\right]\right. \\
& \left.\quad-\frac{1}{4 \pi} \frac{(2 y-1)(2 x-1)}{[y(1-y) x(1-x)]^{1 / 2}}\right\}+O\left(N^{-3 / 2}\right) .
\end{aligned}
$$

One should note that on replacing $x$ by $k_{2} / N$ and $y$ by $k_{1} / N$ in (52) and taking the limit $N \rightarrow \infty,(43)$ is recovered. As was the case for (43), one can also check that (52) remains valid when $N(y-x)=1$ even though it was derived for $N(y-x) \rightarrow \infty$.

\section{Equivalent growth model.}

There is a well-known equivalence between one-dimensional exclusion models and single-step growth models $[10,11,7]$ which in the case of the asymmetric exclusion process may be formulated as follows: the occupancy configuration $\left\{\tau_{1}, ., \tau_{N}\right\}$ represents a surface interface; the presence of a particle $\tau_{i}=1$ represents a decrease in the surface height of one unit at position $i$ whereas $\tau_{i}=0$ represents an increase in surface height of one unit. The surface height $h(k)$ at site $k$ relative to the left hand boundary is then related to number of particles in this region by

$$
h(k)=\sum_{i=1}^{k}\left[1-2 \tau_{i}\right] .
$$

When the total particle number is $N / 2$, so that $h(N)=0$, the two boundaries are at the same absolute height.

The dynamics of the asymmetric exclusion process describes the growth of the surface as follows. When a particle moves from site $i$ to $i+1$ a local minimum of the surface height between sites $i$ and $i+1$ has become a local maximum. A deposition event has thus occurred between $i$ and $i+1$. Deposition at non-boundary sites can only occur if the site is at a lower height than both of its neighbours, whereas deposition at a boundary can occur if the boundary site is at a lower height than its single neighbour. The boundaries can thus be thought of as inhomogeneities in the deposition process $[20,18,21,7]$. The parameters $\alpha$ and $\beta$ represent the deposition acceptance rate at the two boundaries and varying $\alpha$ and $\beta$ corresponds to varying the inhomogeneity in the deposition model. For the case $\alpha=\beta=1$ considered here, 
the two boundaries are on average at the same absolute height, however the bulk is at a lower absolute height. The boundary region of the density profile of the asymmetric exclusion process corresponds to the decrease in the height of the surface from the boundary value, whereas the bulk density profile corresponds to the shallow minimum of the surface height about the middle of the interface. We find on integrating $(42,47)$ that the mean height in the boundary region is

$$
\langle h(k)\rangle=-\frac{2}{\sqrt{\pi}} k^{1 / 2}
$$

and in the bulk is

$$
\langle h(N x)\rangle=-\frac{2}{\sqrt{\pi}}[N x(1-x)]^{1 / 2}
$$

One should note here that the average height at a site in the bulk is governed by the $O\left(N^{-1 / 2}\right)$ corrections to occupancies $1 / 2$ in the asymmetric exclusion model (47). It is thus in the growth model interpretation that the long-range correlations implied by these $O\left(N^{-1 / 2}\right)$ corrections become most significant.

To see this further we shall consider fluctuations in the relative height between different positions on the interface. These fluctuations are related to the fluctuations in particle number between two sites in the asymmetric exclusion model by

$$
\begin{aligned}
& \left\langle[h(j)-h(i)]^{2}\right\rangle-\langle h(j)-h(i)\rangle^{2} \\
& \quad=4\left[\left\langle\left(\sum_{k=i+1}^{j} \tau_{k}\right)^{2}\right\rangle-\left(\sum_{k=i+1}^{j}\left\langle\tau_{k}\right\rangle\right)^{2}\right] \\
& \quad=8 \sum_{k_{2}=i+1}^{j-1} \sum_{k_{1}=k_{2}+1}^{j}\left[\left\langle\tau_{k_{1}} \tau_{k_{2}}\right\rangle-\left\langle\tau_{k_{1}}\right\rangle\left\langle\tau_{k_{2}}\right\rangle\right]+4 \sum_{k=i+1}^{j}\left[\left\langle\tau_{k}\right\rangle-\left\langle\tau_{k}\right\rangle^{2}\right]
\end{aligned}
$$

To determine the fluctuations in particle number in the boundary regions and in the bulk we can integrate (43) and (52) respectively. The task is straightforward if tedious, and we obtain for the boundary region $\left(1 \ll k_{2}<k_{1} \ll N\right)$

$$
\begin{aligned}
& \left\langle\left[h\left(k_{1}\right)-h\left(k_{2}\right)\right]^{2}\right\rangle-\left\langle h\left(k_{1}\right)-h\left(k_{2}\right)\right\rangle^{2} \\
& \quad \simeq-8 \int_{k_{2}}^{k_{1}} d q_{2} \int_{q_{2}}^{k_{1}} d q_{1} \frac{1}{4 \pi\left(q_{1} q_{2}\right)^{1 / 2}}\left[1-\left(1-\frac{q_{2}}{q_{1}}\right)^{1 / 2}\right]+4 \int_{k_{2}}^{k_{1}} d q\left[\frac{1}{4}-\frac{1}{4 \pi q}\right] \\
& \quad=4 k_{2}\left[\lambda\left(\frac{1}{4}-\frac{1}{\pi}\right)-\left(\frac{1}{4}+\frac{1}{\pi}\right)+\frac{2}{\pi} \sqrt{\lambda}-\frac{3}{2 \pi} \sqrt{\lambda-1}+\frac{1}{\pi}\left(\frac{\lambda}{2}+1\right) \arctan (\sqrt{\lambda-1})\right]
\end{aligned}
$$

where $\lambda=k_{1} / k_{2}$, and for the bulk

$$
\begin{aligned}
& \left\langle[h(N y)-h(N x)]^{2}\right\rangle-\langle h(N y)-h(N x)\rangle^{2} \\
& =8 N^{2} \int_{x}^{y} d a \int_{a}^{y} d b\left[\left\langle\tau_{N a} \tau_{N b}\right\rangle-\left\langle\tau_{N a}\right\rangle\left\langle\tau_{N b}\right\rangle\right]+4 N \int_{x}^{y} d a\left[\left\langle\tau_{N a}\right\rangle-\left\langle\tau_{N a}\right\rangle^{2}\right] \\
& \quad+O\left(N^{1 / 2}\right) \\
& =4 N\left\{\frac{1}{4}(y-x)\left[1-\frac{3}{2}(y-x)\right]+\frac{1}{2 \pi}[y-x+3 x(1-y)] \arctan \left[\left(\frac{y-x}{x(1-y)}\right)^{1 / 2}\right]\right. \\
& \left.-\frac{3}{2 \pi}[x(1-y)(y-x)]^{1 / 2}-\frac{1}{\pi}\left([y(1-y)]^{1 / 2}-[x(1-x)]^{1 / 2}\right)^{2}\right\}+O\left(N^{1 / 2}\right) .
\end{aligned}
$$


If we consider the limit of $y-x$ small in (61), which corresponds to two sites well separated microscopically but close to each other on the scale of the interface, then we find that $\left.[h(N y)-h(N x)]^{2}\right\rangle-\langle h(N y)-h(N x)\rangle^{2} \simeq N(y-x)$. This is the result one would obtain from considering the interface as random walk by assuming the steps of the interface to be uncorrelated. Locally, therefore, the correlations do not significantly affect the shape of the interface. However on larger scales the interface is significantly different from a random walk because (55) indicates a curvature of the interface and (52) implies non-trivial fluctuations in the surface height. Thus the apparently weak connected correlations, which in the bulk are of magnitude $O\left(N^{-1}\right)(52)$, accumulate over large scales to lead to a smoothing effect on the interface. This is reminiscent of what has already been found for a Toom interface [22] ( there the smoothing effect was even stronger since the power of $N$ of the height fluctuations was altered by the boundary conditions).

\section{Conclusion.}

To summarise, we have given an exact expression for the 2 point correlation functions of a simple asymmetric exclusion model and have conjectured expressions for all higher correlations. The effect of correlations is highlighted when the model is mapped onto a growth process, for then the leading behaviour is different from mean-field predictions. This implies that long range correlations exist and play an important role in the model. The origin of these long range correlations can be traced back to the boundary conditions. It would be interesting to investigate the effect of different boundary conditions, for example by considering general values of $\alpha$ and $\beta$. To this end an easier route to the exact solution would be most desirable. At the time of finishing the present manuscript, we found with V. Hakim and V. Pasquier an alternative way of writing the steady state which may hopefully give a more direct derivation of the correlation functions and be extended to more general values of $\alpha$ and $\beta$.

In the context of growth, it would be useful to know whether the continuum limits of the height profile and height fluctuations of a microscopic model, as considered in the present work, could be obtained directly from a continuous description, like the KPZ equation [12] with an inhomogeneous deposition at the boundary [20].

Note added in proof: Since completing this work we have found with V. Hakim and V. Pasquier [23] an alternative approach to the problem that allows a proof of the validity of the expressions (15) of the correlation functions conjectured here.

\section{Acknowledgment.}

We thank V. Hakim, C. Kipnis and V. Pasquier for useful discussions. MRE would like to thank the Royal Society for financial support during this work.

\section{Dedication.}

The work of Rammal Rammal in solid state physics and statistical mechanics has been very influential over the last fifteen years. We dedicate this work to his memory. 


\section{Appendix A : recursion for the weights.}

In [8] it was shown that for the model described in the introduction, the weights $f_{N}\left(\tau_{1} . \tau_{N}\right)$ can be calculated explicitly for all $N$. Ref [8] gave the following recursion of the steady state of the weights:

$$
\begin{aligned}
& \text { If } \underline{\tau_{N}=1} \\
& f_{N}\left(\tau_{1}, . \quad \tau_{N-1}, \tau_{N}\right)=\alpha f_{N-1}\left(\tau_{1}, . \quad \tau_{N-1}\right) \\
& \text { If } \tau_{N}=\tau_{N-1}=\ldots=\tau_{p+1}=0 \text { and } \tau_{p}=1 \\
& f_{N}\left(\tau_{1}, . \quad, \tau_{p-1}, 1,0, .0\right)=\alpha \beta\left[f_{N-1}\left(\tau_{1}, . \quad, \tau_{p-1}, 1,0, .0\right)\right. \\
& \left.+f_{N-1}\left(\tau_{1}, ., \tau_{p-1}, 0,0, .0\right)\right] \\
& \text { If } \left.\frac{\tau_{1}=\tau_{2}=\ldots \tau_{N}=0}{f_{N}(0, .0)=\beta f_{N-1}(0, .0}\right) \text {. }
\end{aligned}
$$

This recursion together with the initial conditions

$$
f_{1}(1)=\alpha \text { and } f_{1}(0)=\beta \text {, }
$$

determines the steady state for all system sizes and for all choices of $\alpha$ and $\beta$.

\section{References.}

[1] Liggett T M, Interacting Particle Systems ( NY: Springer Verlag, 1985).

[2] Kutner R, Phys. Lett. 81A, 239 (1981).

[3] van Beijeren H, Kehr K W, Kutner R, Phys. Rev. B28, 5711 (1983).

[4] Katz S, Lebowitz J L, Spohn H, J. Stat. Phys. 34, 497 (1984).

[5] van Beijeren H, Kutner R, Spohn H, Phys. Rev. Lett. 54, 2026 (1985).

[6] Krug J, Phys. Rev. Lett. 67, 1882 (1991).

[7] Janowsky S A and Lebowitz J L, Phys. Rev. A45, 618 (1992).

[8] Derrida B, Domany E, Mukamel D, J. Stat. Phys. 69, 667 (1992).

[9] Gwa L H and Spohn H, Phys. Rev. Lett. 68, 725 (1992).

[10] Meakin P, Ramanlal P, Sander L M, Ball R C, Phys. Rev. A34, 5091 (1986).

[11] Krug J and Spohn H , in Solids far from Equilibrium; Ed Godrèche C (Cambridge UK: CUP 1991).

[12] Kardar M, Parisi G and Zhang Y-C , Phys. Rev. Lett. 56, 889 (1986).

[13] Bak P, Tang C, Wiesenfeld K, Phys. Rev. A38, 364 (1988).

[14] Dhar D, Phys. Rev. Lett. 64, 1613 (1990).

[15] Garrido P L, Lebowitz J L, Maes C, Spohn H, Phys. Rev. A42, 1954 (1990).

[16] Cheng Z, Garrido P L, Lebowitz J L, Valles L, Europhys. Lett. 14, 507 (1991).

[17] Dhar D, Phase Transitions 9, 51 (1987).

[18] Kandel D and Mukamel D, Europhys. Lett. 20, 325 (1992).

[19] Galves A, Kipnis C, Macchioro C, Presutti E, Comm. Math. Phys. 81, 127 (1981).

[20] Wolf D E and Tang L H, Phys. Rev. Lett. 65, 1591 (1990).

[21] Cook J and Wolf D E, J. Phys. A: Math. Gen. 24, L351 (1991).

[22] Derrida B, Lebowitz J L, Speer E R, Spohn H, Phys. Rev. Lett. 67, 165 (1991); J. Phys. A: Math. Gen. 24, 4805 (1991).

[23] Derrida B, Evans M R, Hakim V, Pasquier V, preprint (1993) submitted to J. Phys. A. 